\title{
Wrong Region of Chinese Current Educational Mode and the Analysis of the Education Effect
}

\author{
Yongchun Liu \\ Department of Mechanical and Electrical Engineering, Xiamen University Tan Kah Kee College, Zhangzhou, China
}

Email address:

lyc782@126.com

To cite this article:

Yongchun Liu. Wrong Region of Chinese Current Educational Mode and the Analysis of the Education Effect. Science Journal of Education. Vol. 5, No. 5, 2017, pp. 206-210. doi: 10.11648/j.sjedu.20170505.14

Received: July 5, 2017; Accepted: July 19, 2017; Published: August 11, 2017

\begin{abstract}
Everything in the world has a law, education is also the same. All which conforms to the law are smooth, otherwise, everything is the opposite. In this paper, we used "the basic law curve of life growth education" as a reference, compared and analyzed the existing wrong region of Chinese current educational mode, and also analyzed Chinese current educational effect, also analyzed the social disadvantages caused by the weakening of moral character education, the emphasis and the foothold of different sections of education are emphasized, in this paper we put forward some useful suggestions for the improvement and increases of educational work.
\end{abstract}

Keywords: Basic Law of Education, Moral Character Education, Culture Professional Education, Energy Emotional Input, Education Effect Analysis

\section{Introduction}

The world everything is regular, consistent with the regularity of everything will be smooth, otherwise it will backfire. A year the four seasons is so, three meals a day is also the case. Do not eat meal may be flustered, traffic accidents may occur in violation of traffic, corruption, away from the people, will perish party, let a state perish. Education is an important part of everything, is an important social practice activity to train people. From ancient times to the present, education has never been interrupted, moreover, more and more people pay attention to. Education also has its own regularity, conform to its regularity, conducive to the healthy growth of people, conducive to family well-being, conducive to the stable development of society, it is also conducive to national prosperity. The self regularity of education is "the basic law curve of life growth education" [1]. If education does not conform to its own regularity, it could lead to a series of life, family and social problems. Talking about the regularity of education, everyone will naturally think of the learning phase of primary school, junior high and high school to college, may think this is the law of education. In fact, this understanding is very superficial, this is only a description of the time characteristics of education, it does not involve the essential characteristics of education. The essential feature of education is the two aspects of "moral character education" and "cultural professional education", the proportion of education of these two aspects in different periods of life is not the same. How to grasp the their degrees of the "moral character education" and "cultural professional education" is worth pondering. In this paper, the author takes "the basic law curve of life growth education" [1] as a reference, compared and analyzed the existing wrong region of the current educational mode in China, and also analyzed the current educational effect in China, the emphasis and the foothold of different sections of education are emphasized, in this paper, the author put forward some useful suggestions for the improvement and increases of educational work.

\section{Analysis of the Basic Law of Education}

The author of this paper is in "Research on Mathematical Methods of Basic Laws of Education" [1], the results of other scholars' research on educational law were carefully reviewed and analyzed, from these papers and monographs of "What are the laws of education" [2], "Where to find the law of education" [3], "New exploration on the classification of law of education" [4], "The basic law of education and its application in the research and practice of higher education" [5], "The law of internal and external education, said 'analytic" 
[6], "Pedagogy" [7], "the complexity and nonlinearity of education" [8] as well as the "two basic laws of the external and internal relations of education" [9], which were put forward by professor Pan Maoyuan, a famous educationist in contemporary China, drawing on previous research experience, the author put forward a new idea about the basic law of education in the literature [1], the mathematical function was introduced into the basic law of education, for the first time, the basic laws of education are described in rectangular coordinate system, and to combine the essential characteristics of education with time order organically, the basic law of education is shown in Figure 1, "the basic law curve of life growth education". there are three important function curves in the graph, they are the curves of "moral character education", "cultural professional education" and "energy emotional input".

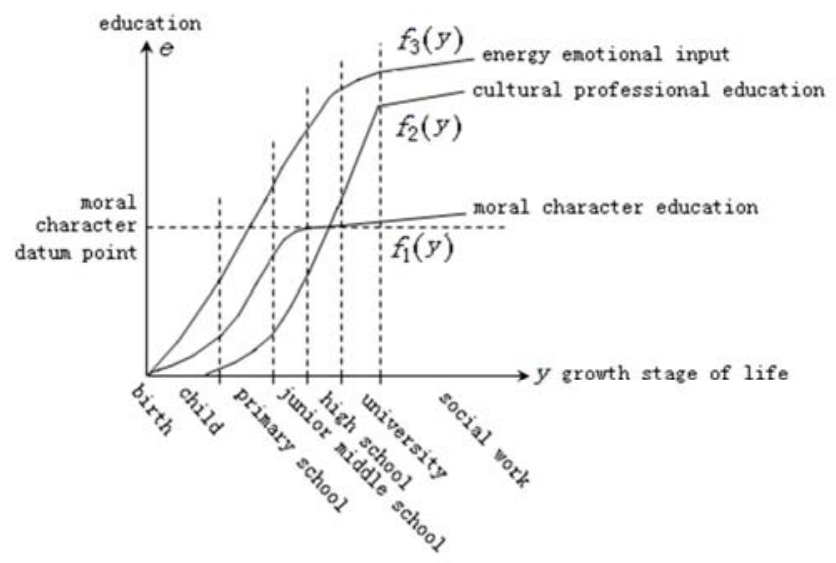

Figure 1. The curve graph of the basic law of life growth education.

The horizontal coordinates $Y$ represents the growth stage of life in Figure 1, the vertical coordinates E represents the education knowledge accepted in the growth phase of life; the curve $f_{1}(y)$ described is the "moral character education" accepted in the growth phase of life, also represents the image perception ability of life; the curve $f_{2}(y)$ described is the "cultural professional education" accepted in the growth phase of life, also represents the logical thinking ability of life; the curve $f_{3}(y)$ described is the "energy emotional input" spent in the process of receiving education of life, also represents the limit of the energy and emotion of life [1].

From Figure 1 we can see that the stage of a person from birth to high school, $f_{1}(y)>f_{2}(y)$, and after that it is $f_{1}(y)<$ $f_{2}(y)$, in the point of second year of senior high school, $f_{1}(y)$ $=f_{2}(y)$. This result tells us, the second year of senior high school is the intersection of "moral character education" and "cultural professional education," is also the datum point of "moral character education". This benchmark shows that when a person comes to senior high school, the moral education must be at a certain height, this is also a person in the long river of life to have the most basic moral character requirements. This law also tells us, before in the second year of high school, we should focus on the education of students on the "moral character education", after that, we should focus on the education of the students on the "cultural professional education". In Figure 1 , the $f_{3}(y)$ function curve shows the the stage of life from the time of birth to the stage of the university is the stage of energy and emotional input, there is no big ups and downs in the whole process, close to linear relation. In other words that the energy and mood are matched with the growth of age in the process of reading and studying. And after from the university to participate in the work the energy and emotions paid to accept education of life becomes much more gentle, this also shows that people's mind becomes mature, quiet, calm a lot. [1] This is the embodiment of the essential characteristics of education in the basic law curve of education, it describes the development and change law of education in the process of life growth intuitively and vividly. Our educational work wants to refer to this basic law to design and planning, the specific content of each stage of education should be close to and comply with the requirements of the basic laws of education.

\section{Wrong Region of Current Educational Mode in China}

China's current education model is mainly a test oriented education model, only fractional theory, high score gotten is a hero. Children begin from kindergarten to learn cultural knowledge course, especially to learn math course, start from the primary school is raised without restriction, not only the more types of course, but also the difficulty is more and more big, to the senior high school almost reaches the ultimate. After a student go home the most something asked by the parents is score, the most concern something of the teacher also is score, the most something of the social propaganda still is score similarly. If according to the author put forward in the literature [1], "the basic law curve of life growth education" as a reference, we can also describe the current education mode of China in the rectangular coordinate system, its intuitive graph is shown in Figure 2.

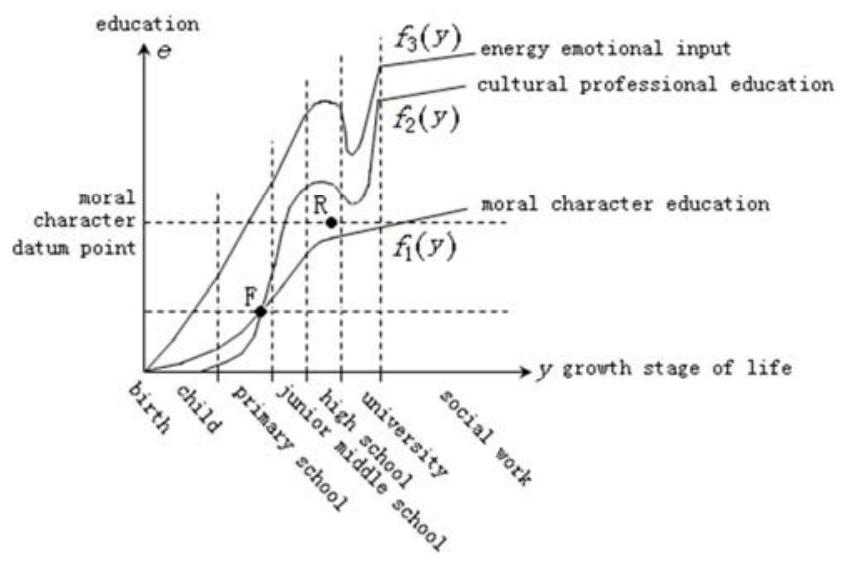

Figure 2. The curve graph of our country's current education mode.

Can be seen from Figure 2, the rising gradient of the function curve of $f_{1}(y)$ is always gentle, this shows that in our country's current education mode, the content of moral character education for students is not enough, the strength is 
relatively weak. But in the period from the beginning of the second year of junior middle school to the third year of university, the function curve $f_{2}(y)$ and $f_{3}(y)$ was appeared a serious bending deformation, in the position of the second and third year of high school was appeared a relative high point, in the position of the first and second year of university was appeared a relative low point, after that they were rose again quickly. This shows that in our current education mode, the content of the "culture professional education" to students is too much, the intensity is also too big. In the period from the beginning of the second year of junior middle school to the third year of high school, the students' learning content and strength almost were reached saturation, the energy and emotion paid by students has been almost reached the limit. After entering the university, although there are many teaching contents of cultural professional courses, but the students' learning energy and emotion were appeared a serious landslide. In the stage of the third and the fourth year of university, although there was some of awakening to the truth, however, it was somewhat late. In the primary school grade five or so, $f_{1}(y)=f_{2}(y)$, after that the function $f_{2}(y)$ is always above of function $f_{1}(y)$. Can see, the datum point of moral character education is moved down from normal $R$ point to $F$ point, This is not to say that people's moral character education in advance to achieve the desired objectives, on the contrary, this is just a pseudo datum point of moral character education. At F point, A person's psychology and intelligence are not perfect yet, the moral character education accepted is also very limited, do not have an independent personality and social work and strain capacity. This can only be explained as that the proportion of "cultural professional education" is much larger than that of "moral character education" after grade five. Obviously, this kind of education mode weakened to the education of the moral character, and strengthened to the education of the cultural professional knowledge, in this mode of education, pupils will be flawed in the psychology and intelligence.

By comparing figure 2 with "the curve graph of the basic law of life growth education" in figure 1, we can see that China's current education mode almost moral character education and culture professional education have the order reversed. According to this education mode, the moral character education has been virtually weakened, even the base point of the moral character education of the students has not been reached, after entering a university also need to make up courses of moral character education. This kind of educational model has obviously and seriously deviated from "the basic law of the life growth education". Much less, a person's energy is limited, If to put too much in one aspect, it is bound to weaken in the other. Junior middle school, in particular, this learning of high intensity in the stage of high school, this education behavior of "trying to help the shoots grow by pulling them upward", its effect is not only not better, but also brings a serious weary and impatient emotion, to cause students' mental distortion, is not conducive to the cultivation and formation of healthy psychology, is not conducive to the improvement of learning ability.
In the basic education stage of life, this weakening to moral character education, and excessive culture professional education, the teaching mode of "trying to help the shoots grow by pulling them upward" is not conducive to the healthy development of the cause of education, is not conducive to the growth of life and social stability. If this kind of educational wrong region is not corrected in time, it will be harmful to the individual, family and society.

\section{Analysis on the Effect of Current Education in China}

Should say, since the reform and opening up, China's education has made rapid development, and has trained a large number of talents for the society. But, everyone should also see, our country in the current education mode, the children from the beginning of kindergarten have already bearing a heavy load, today to have this special class, tomorrow to have also another special class, the parents are also busy awfully. In the primary school, the weight of the bag is close to its own weight of $1 / 3$, homework of every day, if the parents do not help, then almost does not finish, even some math problems let parents also feel a headache. This should be happily growing body, but to be pressed across by a heavy task of learning, many students eyes also begin to myopia, almost lost a happy childhood. From the beginning of junior middle school to high school graduation that is ineffable. Get up earlier than the chicken, sleep later than the dog. You can see from the picture of the college entrance examination shown in figure 3 , the reference book on the desk of the high school grade three is at least half a meter high, the daily homework, practice almost tired students out of breath, the energy and emotion paid for learning is like the spring of the old clock up to the limit. Even so, learning effect is not necessarily good, the parents are anxious to turn round, hate them self to do not help their children.

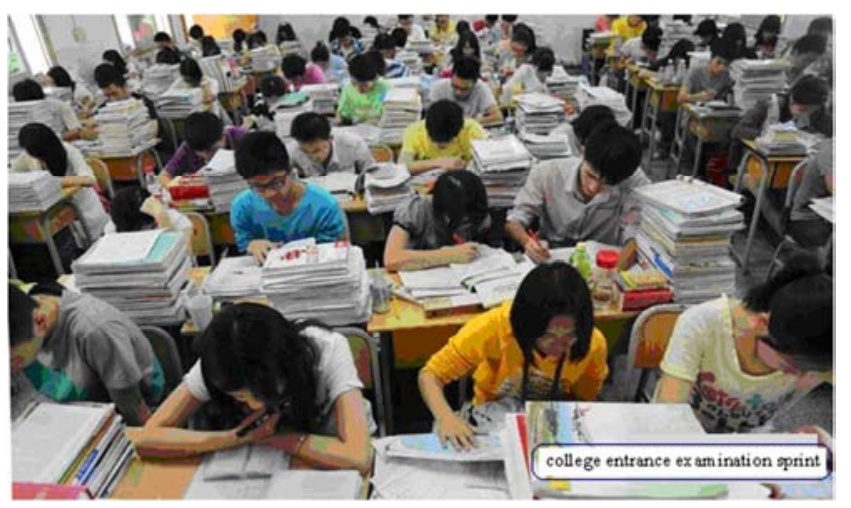

Figure 3. College entrance sprint picture.

From middle school to high school, the life should be the golden period to stimulate imagination, whimsical, discover and cultivate interest, but because of the endless study and homework every day it was mercilessly consumed. No sports, no weekends, the only way to allow students to quickly relax, eliminate mental fatigue and stress is to play the game and 
chase the entertainment star. In the course of time, this way of relaxation has become a very difficult to reverse the habits of life. Such education, learning, seriously compresses the student's curiosity, crowds out their imagination, results a lack of innovation ability, the country's development and the cultivation of high-end technical talents are also missed. Finally get to the end of the college exam, but also lucky to go to college, their pent up mentality finally released like a broken kite, completely relaxed, but there is no the goal of learning, learning momentum can not come up. Almost 1 / 3 of students lost interest in the study of the university, in the class not bring a book, not bring a notebook, not bring a pen, only bring a mobile phone. As can be seen from the photo of the university class shown in figure 4, people who can listen the course carefully is not many, but sleeping, playing mobile phone is the mainstream of the classroom. This is shown in Figure 3 of the college entrance examination sprint stage formed a great contrast, this situation even many university professors feel helpless.

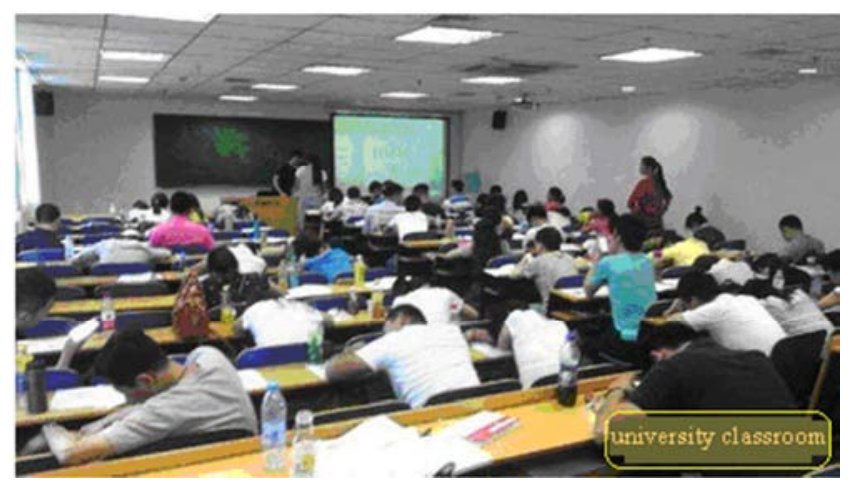

Figure 4. Class status of college students.

Among college students, already about half of the students have been myopia, however, do not concentrate in class, but are keen to play online games, play QQ, play WeChat, listen to music and have fun, no matter how the teacher requirements, are not much of an effect, is almost a numb state. In July 7, 2017, the "Beijing time" (btime.com) Internet portal published an article in the "palm express" column, the title was "Sleeping college students: you are not unemployed, providence will not forgive!" [10], subsequently, the "People's Daily" and "China Youth Daily" WeChat public number also published the article. This article described the actual learning and performance of college students in China from the aspects of "class time", "examination time", "before graduation" and "specialized courses", this kind of unintentional learning, lazy learning attitude really makes people feel very sad. This also further confirms the correctness of the conclusion of the "there are some wrong region of Chinese current educational mode" in this article.

In the "palm express" column published in the "sleeping in college students: you are not unemployed, Providence will not forgive!" [10]'s article,

The effect of this education mode in the society also distraught. People often hear or see students suicide news from different media every year, there was parents to kill their children, there was a student murder, these excesses reflected their psychological vulnerability and lack of emotional binding force. Due to the lack of moral character education, all walks of life are more or less appeared the bad habits of harm others to benefit oneself, official corruption is becoming more and more serious. The old man felled no one dare to help him, nobody dared to talk to strangers, even the long-distance travel or travel and did not dare to communicate with passengers, to see a strange call can not be answered. Not is afraid of being cheated, that is afraid of being calculated, it seems that everything around all fills the horrible trap. To tell the truth, this phenomenon is rarely seen in the past, but now it is not uncommon. In the final analysis, these bad habits are due to the lack of moral character education.

It is gratifying that the Party Central Committee with Chairman $\mathrm{Xi}$ Jinping as the core, earnestly grasps the construction of the working style of the Party, incorruptible government and the fight against corruption, "tigers" and "flies" are been hit together, it has played a very important role for the improvement of the social atmosphere. However, to increase the reform of education model, teaching according to the basic law of education, thus can lay a solid foundation for the improvement of the social atmosphere.

\section{Suggestions on the Improvement of Current Education Mode in China}

In Figure 1, "the basic law curve of life growth education" shown, the life education is divided into three sections: the section from birth to primary school graduation is the section of the physiological and psychological growth education, in this section, the plasticity of the pupils' ideological and moral behavior is the strongest. So, in this section, we must strengthen the education of pupils' moral character, especially family education is very important, parents' behavior is an important object for children to imitate; schools should also increase the proportion and strength of moral character education to conduct to pupils, this is very important, also very precious. The moral education in this section is the moral foundation of a person's life, there is a hundred benefits and there is not any harm. If educational well will be twice the result with half the effort, to educate is not good, may affect a lifetime. Middle school to high school stage is the formation section of logical thinking, but also is the development section of germinating curiosity and creativity. In this section, parents should take children more to contact with nature, more go to Xinhua Bookstore to read some extra-curricular books and science fiction books, school should organize the students to visit the science and technology exhibition and other activities, to lead and inspire children's curiosity and imagination, the innovation and creation for the future and knowledge innovation are foreshadowing. The university is the accumulation section to learn cultural professional knowledge, in this section is mainly to strengthen the learning and accumulation of professional knowledge of science and culture, to lay a solid foundation for the upcoming social work. It is not difficult to see, the emphasis of each section of education is not the same, as long as firmly grasp the these 
characteristics of education, cultivate useful people for the society and sophisticated science and technology talents should be a natural thing.

\section{Conclusion}

Although China's current educational model has achieved remarkable results, for the development of all walks of life to transport a large number of talents, however, to compared with "the basic law curve of life growth education", there are some wrong region of education yet, also produced not a few harmful social impact. Although these effects were limited, but, that already has caused social honesty reducing and the jealousy and lax of people hearts, even there was a crisis of faith. Only by fully understanding the defects of current education in China, according to the basic law of education to develop education, reverse the extreme approach in the process of basic education as soon as possible, to pull the great cause of China's education into the normal track, then, our country's education cause will be more successful.

\section{References}

[1] Yong-Chun Liu. Research on Mathematical Methods of Basic Laws of Education [J]. American Journal of Educational Research, 2017, 5 (3): 251-255.
[2] ZHAO Qingping. What are the Laws of Education [J] China Higher Education, 2012, (18): 1.

[3] FENG Xiangdong. Where to Find the Law of Education [J]. Journal of Higher Education, 2014, (8): 22-29.

[4] CHEN Shaotang. New Exploration on the Classification of Educational law [J]. Journal of Higher Education, 1996, (3): 31-36+91.

[5] PAN Maoyuan. Basic Laws of Education and Its Application in Higher Education Research and Practice [J]. Research in Educational Development, 1997, (2): 3-9.

[6] WAN Jianming. Analysis of "Internal and External Law of Education" [J]. Education Review, 2004, (1): 10-13.

[7] WANG Daojun, WANG Hanlan. Pedagogy [M]. People's Education Press, 1989: 1.

[8] AN Shiao. Complexity and Nonlinear Law of Education [J]. Modern Education Management, 2015, (7): 41-46.

[9] PAN Maoyuan. Basic Laws of Education and Its Application in Educational Research [J]. Jiangsu Education Research, 2009, (4): $3-6$.

[10] Weixin. Sleeping College Students: You are not Unemployed, Providence will not Forgive! [N]. Beijing Time (http://www.btime.com/), 2017-7-7. 